\title{
An Investigation of Self-Interference Reduction Strategy in a Spatially Correlated MIMO Channel
}

\author{
Rosdiadee Nordin \\ Faculty of Engineering and Built Environment, National University of Malaysia, 43600 Bangi, Malaysia \\ Correspondence should be addressed to Rosdiadee Nordin, adee@eng.ukm.my \\ Received 20 May 2011; Accepted 31 October 2011 \\ Academic Editor: Satha K. Sathananthan \\ Copyright () 2012 Rosdiadee Nordin. This is an open access article distributed under the Creative Commons Attribution License, \\ which permits unrestricted use, distribution, and reproduction in any medium, provided the original work is properly cited. \\ One of the efficient ways to transmit high data rate is by employing a multiple-input multiple-output (MIMO) transmission. One \\ of the MIMO schemes, known as spatial multiplexing (SM), relies on the linear independence data streams from different transmit \\ antennas to exploit the capacity from the fading channels. Consequently, SM suffers from the effect of spatial correlation which is \\ the limiting factor in achieving the capacity benefit that SM can offer. In an attempt to increase the robustness of the SM trans- \\ mission in a wide range of correlated channels, the use of dynamic subcarrier allocation (DSA) is investigated. The effective signal- \\ to-interference-and-noise ratio (SINR) metric is used as the performance metric to determine the subcarrier quality which can \\ then be utilised in the allocation. Two novel variants of the subcarrier allocation scheme are proposed. It is shown that the DSA- \\ SINR approach improves the BER performance of SM transmission in highly correlated channels environment.
}

\section{Introduction}

Orthogonal frequency division multiplex (OFDM) is one of the effective mitigation techniques to combat the channel impairments in a wireless network. The multiuser version of OFDM, known as OFDMA, consists of multiplexing different users in the time and frequency domains by assigning subsets of subcarriers to individual users, thus allowing efficient and flexible resource allocation across frequency bands.

OFDMA transmission technology can be further enhanced with the addition of the multiantenna techniques, known as MIMO. There are two popular approaches. The first technique is space-time block code (STBC), proposed by Alamouti [1], which aims to achieve full transmit diversity and reliable communication links. Another scheme, known as spatial multiplexing (SM), introduced by Foschini et al. [2], aims to increase the spectral efficiency by transmitting independent parallel data streams over multiple antennas. SM is commonly implemented in the form of the V-BLAST (Vertical-Bell Laboratories Layered Space-Time) architecture [3].

Foschini and Gans [4] have shown that the channel capacity for a MIMO system increases as the number of antennas increases and is proportional to the number of minimum transmit and receive antennas. However, the multiplexing gain is dependent on the number of transmit-receive antennas, which are subjected to uncorrelated fading. In other words, the spectral efficiency that can be exploited in a MIMO scheme depends strongly on the statistical behaviour of the spatial fading correlation, also described by Gesbert et al. [5] as the effect of self-interference. This effect can be illustrated in Figure 1, whereby spatial subchannel 1 from $T_{2}$ acts as an interferer for the desired signal to be transmitted between $T_{1}$ to $R_{1}$, and the same occurs from the $T_{2}$ and $R_{2}$ antennas.

SM schemes rely on the linear independence between the channel responses corresponding to each pair of transmitreceive antennas. As the spatial correlation increases, crosscorrelation occurs between the spatial multiplexing data streams. Consequently, SM schemes suffer considerably from spatial correlation, resulting in ill-conditioned matrices, which can cause degradation of system capacity.

Several factors determine the degree of spatial correlation, such as antenna element spacing [6], separation between transmitter and receiver [7, 8], array orientation [9], and multipath angular spread [10]. As the channel achieves full correlation, the effective capacity gain of a MIMO channel is similar to that of a SISO system, thus diminished 


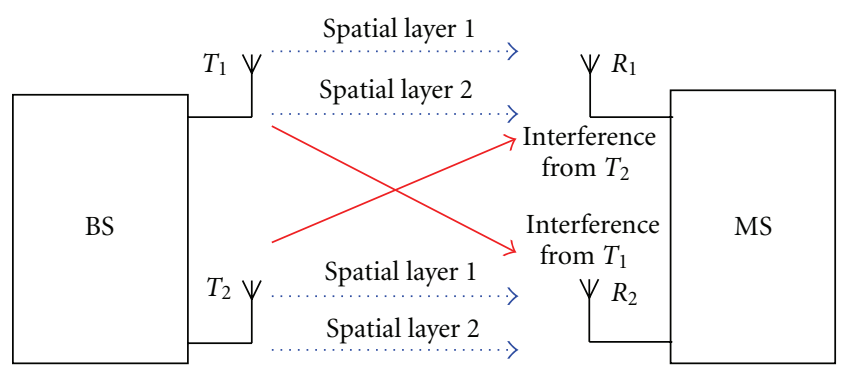

FIGURE 1: Example of self-interference in a $2 \times 2$ SM transmission.

the capacity benefit offered by MIMO system if the effect of self-interference is not properly mitigated.

In practice, the spatial subchannels between different antennas are often correlated and therefore the full potential multiantenna gains may not always be obtainable. However, the received signal may still have a strong spatial signature in the sense that stronger average signal gains are received from certain spatial directions. In this paper, two novel subcarrier allocation schemes are proposed. Both variants of the subcarrier allocation scheme take advantage of independent channel variations across users to improve the network performance through frequency and spatial diversity. The performance of the proposed algorithm is analyzed when operating in different correlated channel environments.

The rest of the paper is organised as follows. The problem formulation for resource allocation in OFDMA networks is presented in Section 2. In Section 3, system setup and simulation parameters are presented. In Section 4 , the performance of the proposed algorithm is further investigated and analysed in different correlation environments. Section 5 provides the conclusions drawn from this paper, whereby important analysis and research findings will be summarized and presented.

\section{Self-Interference Mitigation Strategy}

2.1. SINR Metric. In this section, a mathematical definition of the SINR metric is presented. The proposed subcarrier allocation uses the SINR as a performance metric to determine the subcarrier quality for allocation purposes. SINR metric has knowledge of the channel quality at every subcarrier level, whereby it indicates the spatial information and self-interference caused by the mismatch between the spatial subchannels. The mathematical model for the received signal in a MIMO-OFDMA system, after FFT and guard removal, is described as follows:

$$
Y_{u}^{s}=H_{u}^{s} X_{t}^{s}+N_{u}^{s}
$$

where the subscript $u$ denotes the MS index, $s$ denotes the subcarrier index, and $H_{u}^{s}$ is the channel matrix containing the MS $u$ frequency responses of the channels between $N_{t}$ transmit and $N_{r}$ receive antennas at subcarrier $s$ and applied to the subcarriers of the OFDMA signal on a cluster basis, while $N_{u}^{s}$ denotes AWGN noise and $X_{t}^{s}$ denotes $N_{t} \times 1$ matrix containing transmit signals. At the receiver, the OFDMA system adopts a linear MMSE configuration:

$$
G_{u}^{s}=\left(\left(H_{u}^{s}\right)^{H} H_{u}^{s}+\mathrm{SNR}^{-1} I\right)\left(H_{u}^{s}\right)^{-1} .
$$

The MMSE filter has the ability to mitigate self-interference whilst not adversely amplifying the received noise. The MMSE filter is also able to separate the spatial subchannels of the MIMO structure [11]. As a result, different spatial subchannels can be allocated to different users to achieve additional spatial multiuser diversity gain. On the other hand, this typically increases the amount of feedback by the number of spatial subchannels. The received signal is multiplied by an MMSE filter given by (2)

$$
G_{u}^{s} Y_{u}^{s}=G_{u}^{s} Y_{u}^{s} X_{t}^{s}+G_{u}^{s} N_{u}^{s} .
$$

The receiver then calculates the effective SINR (SINR) per subcarrier and feeds back that information to the transmitter. Effective SINR stands for the SINR per subcarrier, obtained by linearly combining the signals from all the received antennas. The SINR metric captures the variation of the SINR in the subcarrier domain. To calculate the effective SINR, the postdetection SINR for each subcarrier needs to be calculated after the MIMO processing. The MS $u$ computes the SINR at every spatial subchannel for every subcarrier [11] (the subcarrier index, $s$, is omitted for simplicity of presentation):

$$
\operatorname{SINR}_{u}^{q}=\frac{\left|\left(G_{u} H_{u}\right)_{q q}\right|^{2} \varepsilon_{s}}{\left|\left(G_{u} H_{u}\right)_{q j, j \neq q}\right|^{2} \varepsilon_{s}+\left(\left|G_{u}\right|_{q q}^{2}+\sum_{j \neq q}\left|G_{u}\right|_{q j}^{2}\right) N},
$$

where $q$ is the spatial subchannel considered in the allocation algorithm. In the case of SISO system, $q=Q=N_{r}=1$. $\varepsilon_{s}$ denotes the average symbol energy and $|\cdot|_{q j}$ denotes the element located in row $q$ and column $j$. The SINR metric aims to compute self-interference with knowledge from the data stream component, $|\cdot|_{q q}$, and self-interference component, $|\cdot|_{q j, j \neq q}$, from the adjacent transmitted data streams, also known as spatial interferer. For example, in a $2 \times 2 \mathrm{MIMO}$ system $(q=2), h_{11}$ and $h_{22}$ are the data stream components, while $h_{12}$ and $h_{21}$ are considered as spatial interferers to channels $h_{11}$ and $h_{22}$, respectively.

MS $u$ calculates the SINR metric across all subcarriers in each cluster and sends this to the BS via the feedback channel. This requires a large amount of feedback information. However, this paper does not consider a feedback reduction scheme although there are several works to reduce the required feedback in schemes. Thus, a full SINR feedback scheme that is free from error is assumed.

2.2. DSA Scheme to Combat Channel Correlation. In an OFDMA system with users fading independently, there is likely to be a user with a good channel at any given time slot/subcarrier. By scheduling transmission so that users transmit at times and frequency resources when their channel conditions are favourable, significant gains from the effective channel can be achieved. So if a deterministic rather than random allocation of subcarriers is employed, the 


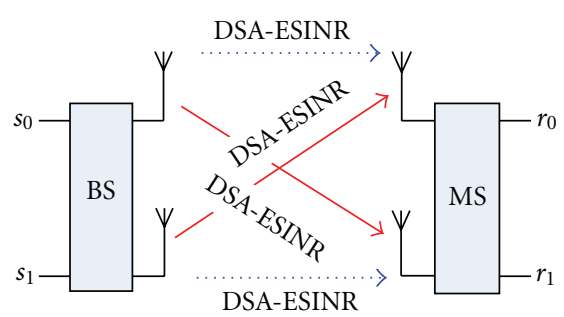

(a) "All SINR" variation

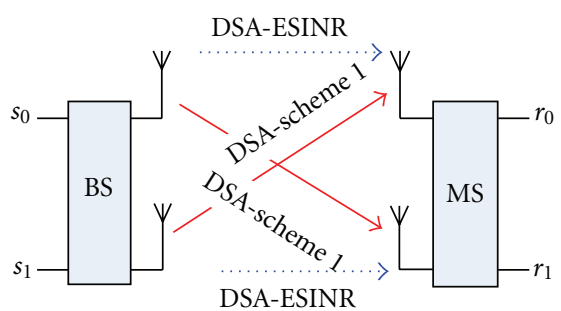

(b) "Partial SINR" variation

FIgURE 2: Variations of the proposed DSA-SINR algorithm.

multiuser diversity can be exploited to ensure that most users can be allocated the best subcarriers for them with minimal clashes. As a result, bit error rate (BER) can be improved by serving the user with the strongest channel.

Initially, DSA work has focussed on SISO systems [12]. Subsequently, the research focus has been expanded to MIMO schemes by Peng [13], whereby several variations of the algorithm (referred to as "schemes") were introduced. Each scheme corresponds to different spatial correlation condition. Channel gain was used as a metric to determine the subcarrier allocation process. The first scheme, referred to as DSA-Scheme 1, performed subcarrier allocation separately for each spatial subchannel and it takes no relation of one spatial subchannel to the other adjacent subchannel. An extension to DSA-Scheme 1, known as DSA-Scheme 5, aims to combat the effect of self-interference in a correlated environment. This scheme considers the previously allocated subcarriers from the previous spatial subchannel by means of iteration. The number of near adjacent subcarriers avoided is measured by parameter $l$. From the simulation results, it is shown that, when $l=10$, significantly enhanced BER gain can be obtained in a fully correlated environment.

The proposed DSA-SINR algorithm is an alternative approach to the subcarrier allocation procedure to work in [13]. In this paper, there are two variations of the proposed DSA-SINR subcarrier allocation, namely, "All SINR" and "Partial SINR", as depicted in Figure 2. Instead of using the channel gain as the performance metric, "All SINR" scheme uses the SINR metric as the performance metric to determine the subcarrier allocation in the same manner on every spatial subchannel.

As with the "All SINR" scheme, the dynamic subcarrier allocation is applied independently across spatial subchannels in "Partial SINR" scheme. However, "Partial SINR" used two different performance metrics to determine the subcarrier allocation at each of the spatial subchannels: (i) at the parallel subchannels, SINR metric is used to identify the subcarriers that are affected by self-interference, and (ii) the spatial interferers use the channel gain as a metric to determine the subcarrier allocation, which is similar to the DSAScheme 1. By doing the allocations in this manner, users are prevented from sharing the subcarriers with the spatial interferer, thus helping to avoid the use of an extra coding scheme and reducing the complexity of the algorithm. The combination of different performance metrics in "Partial SINR" is expected to reduce spatial correlation, whilst still seeking a maximal allocation of channel energy.
This also ensures that spatial diversity cannot be lost due to highly correlated spatial subchannels. In both variations of the allocation scheme, each of the spatial subchannels will be allocated with different sets of subcarriers. In the SISO case, Jang and Lee [14] show that the capacity can be maximized if a subcarrier is only assigned to one user, as it helps to reduce the interference from other users signals that happen to share the same subcarrier. This paper extends the theory presented by Jang and Lee [15] into the MIMO case, where the number of users sharing the same subcarrier is limited to the number of available spatial subchannels (two spatial subchannels in this case). The algorithm is iterated periodically, and once a frequency-space resource is allocated to a user, it is reserved for that user until the algorithm is reiterated to change the allocation, that is, no further allocation to that particular user.

Before the allocation takes place, the $u$ th MS computes the MMSE filter, (2) and multiplies the received signal by $G_{k}$, (3). The $u$ th mobile station then computes the SINR and channel gain of the qth spatial subchannel (4). The $u$ th MS then sends them back through the feedback channel to the base station. The base station uses the feedback information as an input for the DSA.

The nomenclatures are set first as references. In the following algorithm, $q=\{1, \ldots, Q\}$ represents spatial subchannel considered for the allocation algorithm. $P_{u}^{q}$ and $E_{u}^{q}$ represent the average received power gain and SINR metric for user $u$ at the $q$ th spatial subchannel, $U$ is the total number of users, and $S$ is a $Q$ by $N_{q}$ matrix where each row is a vector containing the indices of the useable subcarriers for the particular spatial subchannel, that is, $N_{q}=\left\{1, \ldots, N_{\text {sub }}\right\}$, where $N_{\text {sub }}$ is the total number of useable subcarriers. The $h_{u, s}^{q}$ and $\operatorname{SINR}_{k, s}^{q}$ are the channel response and SINR metric for user $u$ at subcarrier $s$ and spatial subchannel $q$, and $\mathbf{C}_{s, u}$ is a matrix to store the subcarrier indices (subcarrier location) of the allocated subcarriers for user $u$ and subcarrier $s$.

The "Partial SINR" variation of the DSA-SINR algorithm can be described as follows:

(1) Initialization

set $\mathbf{P}_{u, q}=0$ for all users, $u=1, \ldots, U$; Set $\mathbf{C}_{u, s, q}=0$ for all users $u=1, \ldots, U$ and spatial subchannel $q=$ $\{1,2, \ldots, Q\}$; set $s=1$.

(2) Main Process While $N_{q} \neq 0_{N_{\text {sub }}}$

\{ (a) Generate short list of users start with user with small SINR metric and channel gain. For the first 
iteration, assume all users have equal data rate as no subcarriers have been allocated; hence, the list may be entirely arbitrary. Find user $u$ satisfying

$$
\begin{aligned}
& \text { Main : } E_{u}^{q} \leq E_{i}^{q}, \\
& \text { Interferer : } P_{u}^{q} \leq P_{i}^{q}, \quad \forall i, 1 \leq i \leq U .
\end{aligned}
$$

(b) For the user $u$ in (a), find subcarrier $s$ satisfying

$$
\begin{aligned}
& \text { Main : } \operatorname{SINR}_{u, s}^{q} \geq \operatorname{SINR}_{u, j}^{q}, \\
& \text { Interferer : }\left|h_{u, s}^{q}\right| \geq\left|h_{j, s}^{q}\right|, \quad \forall j \in S .
\end{aligned}
$$

(c) Update $\operatorname{SINR}_{u, s}^{q}, P_{u}^{q}, N_{q}$, and $\mathrm{C}_{s, u}$ with $u$ and $s$ in (b) according to

$$
\begin{aligned}
& E_{u}^{q}=E_{u}^{q}+\operatorname{ESINR}_{u, s}^{q} \text { (main subchannel), } \\
& P_{u}^{q}=P_{u}^{q}+\left|h_{u, s}^{q}\right|^{2} \text { (interferer subchannel), } \\
& N_{q}=N_{q}-n \\
& \mathrm{C}_{s, u}=n \\
& s=s+1
\end{aligned}
$$

where $\mathrm{C}_{s, u}$ is the allocation matrix to record the allocated subcarrier, $s$ for user $u$.

(d) Go to the next user in the short list in (a), and repeat (b) to (c) until all users are allocated another subcarrier, $N_{q} \neq 0$. $\}$

For the "All SINR" variation, the allocation involves replacing the channel gain to SINR metric as the performance metric at the spatial interferer. In other words, spatial interferer performed similar subcarrier allocation to the main parallel channel.

The algorithm ranks users from the lowest to the highest SINR metric at each main spatial subchannel. Consequently, the next best subcarriers are allocated to users in rank order, allowing users with the lowest SINR, that is, users that suffer from "severe" self-interference effect or poor received signal at that particular spatial subchannel, to have the next best subcarrier with the highest SINR metric that is available for the next transmission.

\section{Simulation Environment and Parameters}

3.1. SM-OFDMA Design Parameters. The simulation is performed in an SM-OFDMA environment. Different schemes of modulation such as binary phase shift keying (BPSK), quadrature phase shift keying (QPSK), and quadrature Amplitude Modulation (QAM) can be applied.

Modulation type affects both the data capacity in a given channel and the robustness with regard to noise and interference. The OFDM parameters and six different modulation and coding schemes (MCSs) are summarised in Tables 1 and 2 and used throughout this paper. The ideal wireless networks are characterised by their ability to operate in low
TABle 1: Physical layer parameter for OFDM system.

\begin{tabular}{lc}
\hline Parameter & Value \\
\hline Operating frequency & $5 \mathrm{GHz}$ \\
Available bandwidth $(\mathrm{BW})$ & $100 \mathrm{MHz}$ \\
Transmit information duration $\left(T_{\mathrm{tx}}\right)$ & $10 \mathrm{~ns}$ \\
FFT size $\left(N_{\mathrm{FFT}}\right)$ & 1024 \\
Useable subcarriers $\left(N_{\text {sub }}\right)$ & 768 \\
Subcarrier spacing $\left(\Delta_{f}\right)$ & $97.66 \mathrm{kHz}$ \\
Useful symbol duration $(T)$ & $10.24 \mu \mathrm{s}$ \\
Guard interval $(\mathrm{GI})$ & 176 \\
Total symbol duration $\left(T_{s}\right)$ & $12.00 \mu \mathrm{s}$ \\
\hline
\end{tabular}

SNR conditions but still offering high capacity with efficient spectrum utilisation. Therefore, a suitable channel model is considered to investigate the appropriate system. The channel is adopted from SCM "Urban Micro" [15] standard.

"Urban Micro" environment represents a very small cell in an ultrahigh-density urban area with cell radius of approximately less than $500 \mathrm{~m}$ and BS antennas located at rooftop level. The MIMO channel model is simulated in a fixed channel matrix so that both receiver and transmitter are static with respect to each other and the path loss remains approximately constant during the measurement duration. The key parameters for the channel model are summarised in Table 3. It is also observed that the channel coherent bandwidth is about $3.98 \mathrm{MHz}$, which is much less than the available bandwidth for the channel model, thus conformed that it is a wideband channel for this considered OFDM system.

A packet size of 54 bytes is considered throughout this paper. In addition, 2000 i.i.d. quasistatic Rayleigh distributed channel samples per OFDM symbol are used in each simulation to achieve stable averaging over wide fading channel condition. 16 users are considered to exploit the multiuser diversity gain with a total of 768 useable subcarriers to be equally shared among the users with FFT size, $N_{\mathrm{FFT}}=1024$. A $2 \times 2$ antenna configuration is used for the SM scheme. It is assumed that the BS has perfect knowledge of the channel transfer function for those subcarriers that have been allocated to it and this is later used for equalisation and decoding purposes.

3.2. Correlated Channel Models. The main aim of this paper is to investigate the SM performance at different levels of spatial correlation: from ideal to extreme correlation scenarios. In the ideal case, the channel is uncorrelated and the effect of self-interference is very minimal, while "fully" correlated channel represents the worst case scenario, whereby the effective capacity gain is equal to that of a SISO system. The spatial correlation matrix of the MIMO channel, $R_{\mathrm{MIMO}}$, is the Kronecker product of the channel matrix between BS and MS, $R_{\mathrm{MIMO}}=R_{\mathrm{MS}} \otimes R_{\mathrm{BS}}$ as proposed by Kermoal et al. [16]. The proposed correlation scenarios as summarised in Table 4 . The correlation cases are derived based on practical wireless channel measurements from previous works as published in [17-19]. 
TABLE 2: Modulation and coding schemes (MCS) for the MIMO-OFDMA system.

\begin{tabular}{|c|c|c|c|c|c|c|}
\hline Modes & 1 & 2 & 3 & 4 & 5 & 6 \\
\hline Modulation & BPSK & QPSK & QPSK & 16-QAM & 16-QAM & 64-QAM \\
\hline Coding rate & $1 / 2$ & $1 / 2$ & $3 / 4$ & $1 / 2$ & $3 / 4$ & $3 / 4$ \\
\hline Data bits per OFDM symbol & 768 & 1536 & 2304 & 3072 & 4608 & 6912 \\
\hline Coded bits per OFDM symbol & 1536 & 3072 & 3072 & 6144 & 6144 & 9216 \\
\hline Total bit per OFDM symbol & 1024 & 2048 & 3072 & 4096 & 6144 & 9216 \\
\hline Nominal bit rate [Mbps] & 64 & 128 & 192 & 256 & 384 & 576 \\
\hline Total bit rate [Mbps] & 85.33 & 170.67 & 256.00 & 341.33 & 256.0 & 339.00 \\
\hline
\end{tabular}

TABle 3: "Urban Micro" channel model parameters.

\begin{tabular}{lc}
\hline Parameters & SCM Urban Micro \\
\hline Bandwidth & $5 \mathrm{MHz}$ \\
Excess delay spread & $923 \mathrm{~ns}$ \\
Mean delay spread & $251 \mathrm{~ns}$ \\
Carrier frequency & $2 \mathrm{GHz}$ \\
\hline
\end{tabular}

TABle 4: Correlation modes and their coefficient.

\begin{tabular}{lcc}
\hline \multirow{2}{*}{ Correlation modes } & \multicolumn{2}{c}{ Correlation coefficient } \\
& $R_{\mathrm{BS}}$ & $R_{\mathrm{MS}}$ \\
\hline "Full" & 0.99 & 0.99 \\
Uncorrelated & 0.00 & 0.00 \\
\hline
\end{tabular}

Figure 3 shows all the spatial subchannels in the uncorrelated $2 \times 2$ MIMO channel. It is observed that each spatial subchannel is randomly distributed across the subcarriers, which suggests that there is negligible influence from the other transmitting spatial subchannels (interferer) during the transmission. Figure 4 is the example of the channel gain when it suffers from "fully" correlated channel. It can be observed that the spectral shape and amplitude of channel gain at every spatial subchannel is almost identical. This is when the effect of self-interference dominates the MIMO performance and the effective channel is similar to SISO.

3.3. Uncorrelated Channel: "Default" versus "Forced". In this paper, uncorrelated channels are organised in two categories: "Default" and "Forced." "Default" uncorrelated channel is generated from the default parameters of the representative channel model, while the "Forced" uncorrelated channel is generated by using the Kronecker product [16], whereby the channel is forced to have a correlation coefficient of 0.00 . Table 5 compares the average correlation coefficient between default and forced uncorrelated channel models before the allocation takes place.

From the table, the correlation coefficients of default channels are higher than "Forced" channels; however, the correlation coefficient is considered at a moderate level and is acceptable in practical implementation as a MIMO channel is expected to generate some amount of spatial correlation, especially in a populated urban environment.
TABLE 5: Correlation coefficient measurement for uncorrelated channel.

\begin{tabular}{lcc}
\hline Correlation coefficient & $h_{11}$ and $h_{12}$ & $h_{21}$ and $h_{22}$ \\
\hline Default & 0.446 & 0.320 \\
"Forced" & 0.000 & 0.000 \\
\hline
\end{tabular}

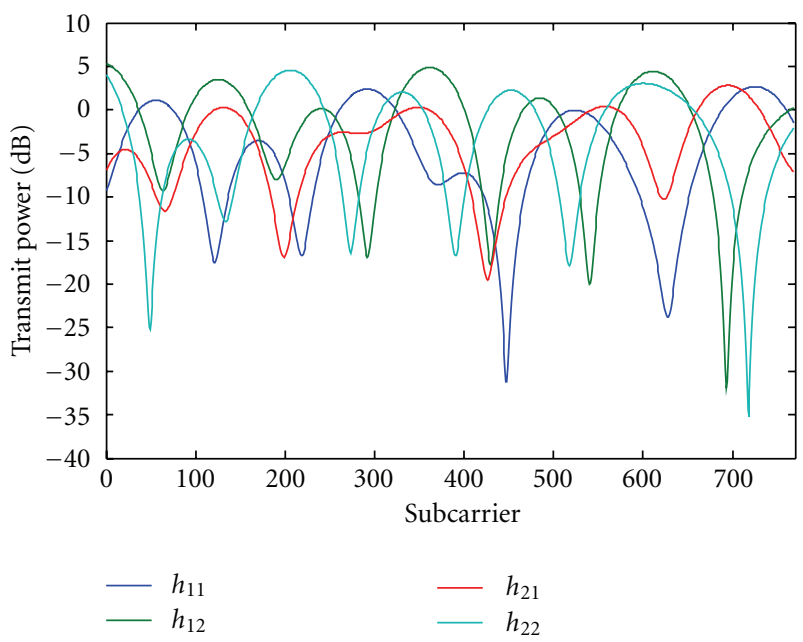

FIGURE 3: Channel gain at every spatial subchannel (uncorrelated channel).

\section{Simulation Results}

4.1. Comparison between Two DSA-SINR Variations. There are two variations of the proposed DSA-SINR schemes: (i) all spatial subchannels utilising DSA-SINR scheme (here referred to as "All SINR") and (ii) parallel spatial subchannel allocated using DSA-SINR scheme, while the "interferer" is allocated with channel gain (referred to as "Partial SINR"). The main aim of this section is to illustrate the advantages and disadvantages of both variations of the allocation scheme.

The result from Figure 5 shows that the "Partial SINR" scheme has better advantages in terms of BER gain, compared to "All SINR" scheme when simulated in a "Default" uncorrelated channel. This can be related to the earlier result in Table 5, where it is shown that the default uncorrelated channel model carries some degree of self-interference, resulting in loss of BER gain. 


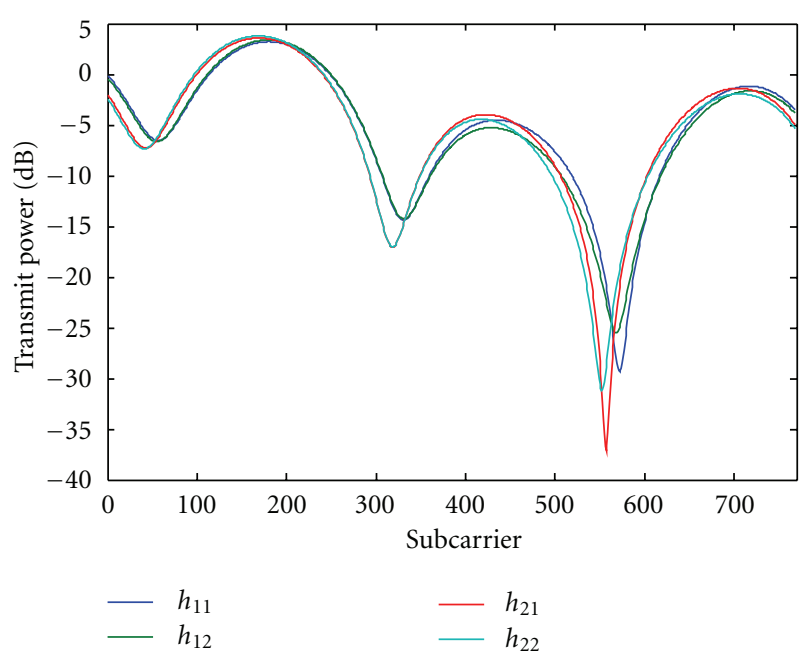

FIgURE 4: Channel gain at every spatial subchannel ("Full” correlation mode).

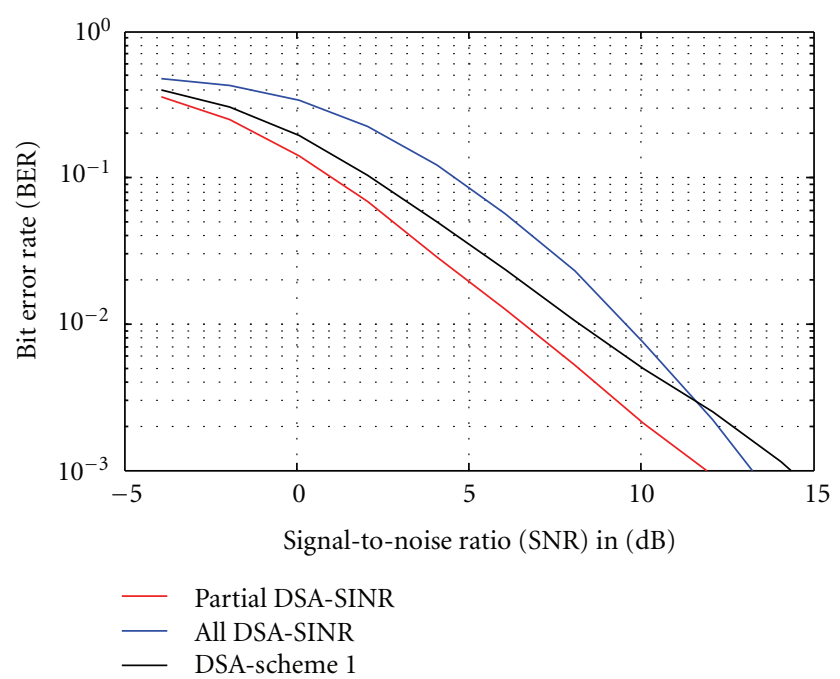

FIGURe 5: BER comparison between "Partial SINR" and "All SINR" schemes in a "default" uncorrelated channel.

When simulated in a "fully" correlated channel different correlation cases, as shown in Figure 6, it can be seen that the "All SINR" scheme has poor BER performance compared to the "Partial SINR" scheme, whereby, at BER $=10^{-3}$, the "Partial SINR" scheme has an advantage of approximately $3 \mathrm{~dB}$ in the "Full" correlation case, while there is minimal margin of BER improvement for the uncorrelated channel.

The dynamic combination between SINR metric in parallel channels and channel gain at spatial interferers makes the "Partial SINR" scheme a more attractive candidate to combat the debilitating effect of self-interference, compared to the "All SINR" scheme.

This can be explained by observing the allocated subcarriers for a user, at a particular time sample in a fully correlated channel between one of the parallel channels and its adjacent spatial interferer. Figure 7 shows the allocated subcarriers for the "All SINR" scheme. In this scheme, the

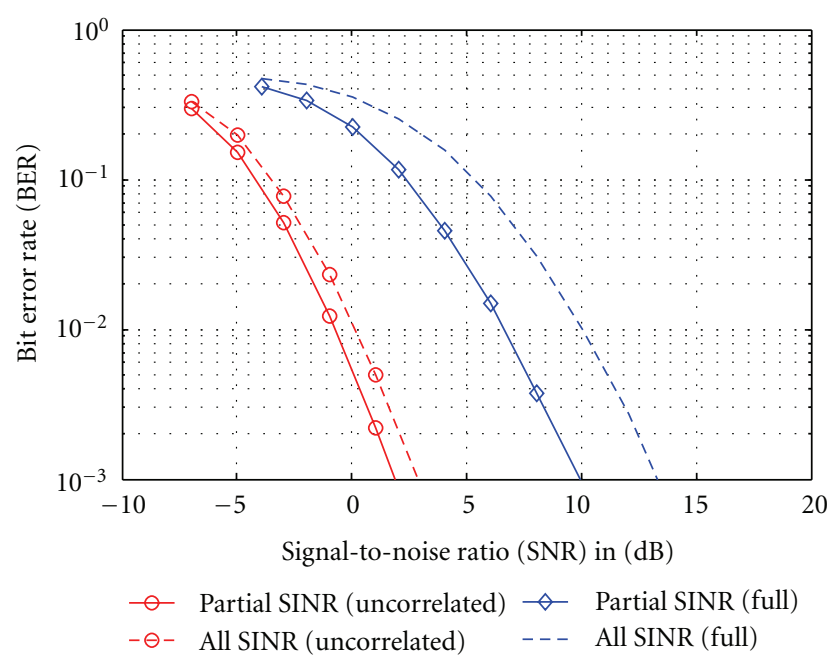

FIGURE 6: BER performance between two variants of DSA-SINR in different correlation modes.

allocated subcarriers at the spatial interferer are almost the same as those allocated in the parallel channel (source), which causes the scheme to be unable to differentiate between subcarriers that are severely affected by self-interference and those subcarriers that still can offer good channel quality in a fully correlated channel.

It can be observed that SINR metrics for both spatial subchannels are almost identical to each other, and since the allocation relies on the SINR metric, both the spatial subchannels allocate the subcarriers when the SINR metric is at its peak. But since each of the spatial subchannels has knowledge on the level of self-interference from the other spatial subchannel, both of the spatial subchannels eventually will share the same subcarrier indexes, resulting in poor spatial diversity gain. The variation of the allocated subcarrier is also wider across the subcarriers range, which creates diverse channel quality across the subcarrier range, but the allocation avoids the selection of subcarriers with high channel gain, resulting in poor BER performance compared to the "Partial SINR" scheme.

In the "Partial SINR" scheme, as shown in Figure 8, the DSA-SINR algorithm at the parallel channel avoids the selection of similar subcarriers that are affected by self-interference while not compromising the best channel quality given by DSA-Scheme 1 at the interfering channel. This results in improved BER performance, as shown in Figure 6.

Other than that, the "Partial SINR" scheme has lower complexity compared to the "All SINR" scheme since the "Partial SINR" scheme only uses the spatial parallel subchannels to determine the self-interference caused by the spatial interferers, while the "All SINR" scheme needs to perform the arithmetic to calculate the SINR metric at all spatial subchannels. In other words, the "Partial SINR" offers lower allocation complexity with extra BER gain compared to the "All SINR." For the remaining part of this paper, the "Partial SINR" algorithm will be considered exclusively and the term "DSA-SINR algorithm" will be used to refer to the "Partial SINR" variant. 


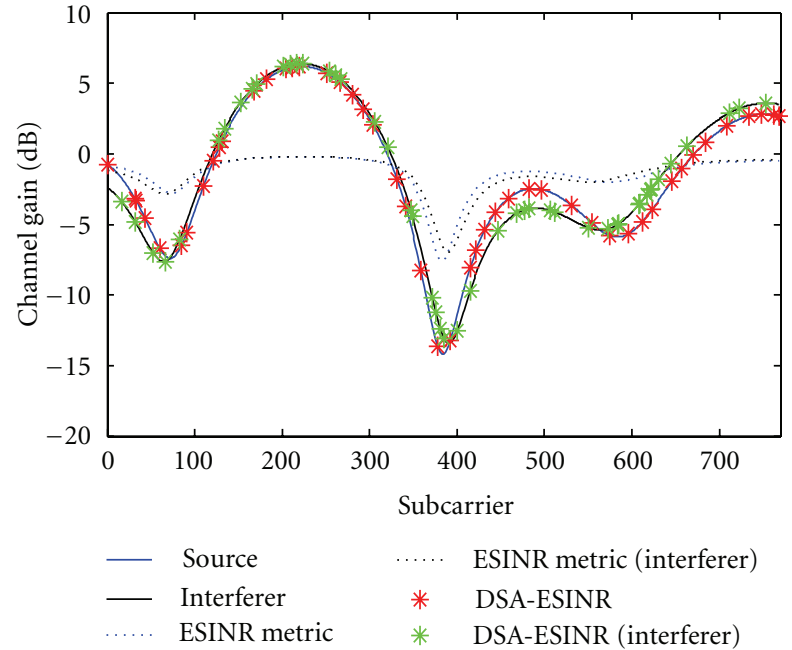

FIGURE 7: Example of subcarrier allocation in a "fully" correlated channel based on "All SINR" scheme.

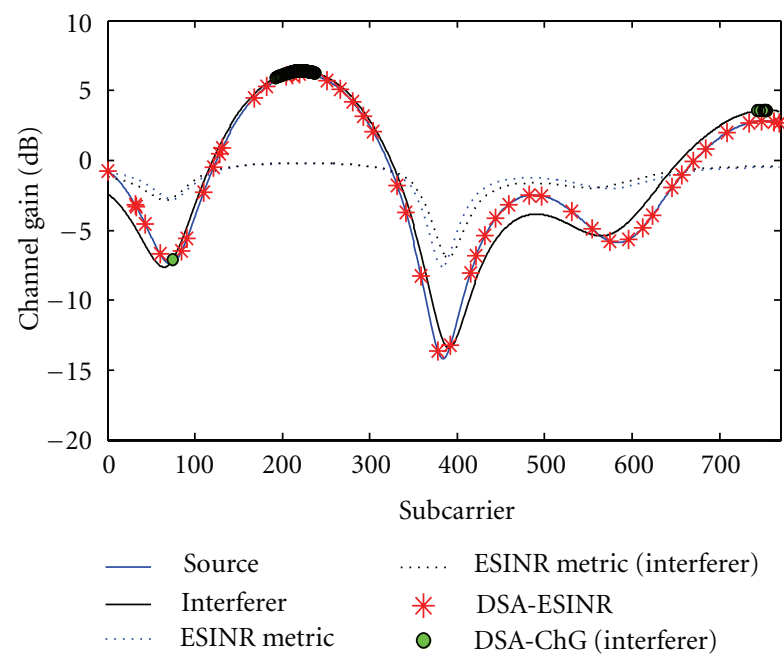

Figure 8: Example of subcarrier allocation in a "fully" correlated channel based on "Partial SINR" scheme.

4.2. Performance in Uncorrelated Channel. Figure 9 compares the BER performance between both types of uncorrelated channel under QPSK, 1/2 rate MCS. BER performance in AWGN channel is plotted as a reference. The uncorrelated channel generated from the default channel model parameters is found to suffer from some BER loss, compared to the "Forced" uncorrelated channel. This conforms to earlier findings in Table 5, which shows that self-interference exists in the "Default" channel model but the effect is also confirmed to be minimal. The curve trend also suggests that the "Forced" uncorrelated channel has a similar trend to that of the Gaussian curve, which suggests that the influence of spatial correlation has been eliminated from "Forced" uncorrelated channel, thus allowing the BER performance to achieve approximately $2 \mathrm{~dB}$ towards AWGN channel. However, to achieve a "Forced" uncorrelated channel in practical

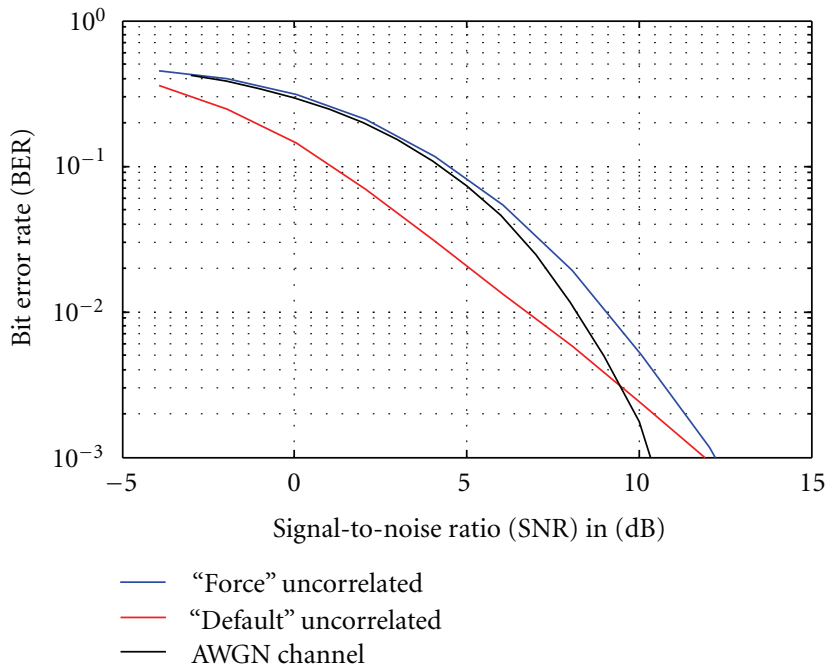

FIGURE 9: BER performance comparison between default and "forced" uncorrelated channels.

wireless implementation is a very challenging task. For the remainder of this paper, the uncorrelated channel generated from the default channel model parameters is used as a representation of a MIMO channel model in realistic wireless environment.

Figure 10 compares BER performance for DSA-SINR and DSA-Scheme 1 in a "Forced" uncorrelated channel. It can be seen that both DSA-SINR and DSA-Scheme 1 share almost similar BER performance in the "Forced" uncorrelated channel. This is because the presence of self-interference is negligible; thus there is no correlation between subcarriers in parallel channel and spatial interferers, resulting in similar BER performance for both allocation schemes. This is not beneficial to exploit the knowledge of spatial interference when the channel is naturally uncorrelated. Another observation is the smooth degradation of BER performance as the MCS increases. This is expected since higher modulation order is more sensitive towards the effect of channel imperfection, especially ISI, which dominated the channel for the SCM "Urban Micro" case.

The DSA-SINR performance is better than the DSAScheme 1 in the "Default" uncorrelated channel, as shown in Figure 11. This can be explained from the correlation coefficient for both types of uncorrelated channel, which is shown in Table 5. The correlation coefficient for SCM "Default" channel model is higher than that for "Forced" uncorrelated, as a result, DSA-SINR algorithm attempts to minimise the spatial correlation within the channel resulting in improved BER gain compared to DSA-Scheme 1. This result also shows the ability of the DSA-SINR algorithm to mitigate the effect of self-interference based on the correlation coefficient of a particular pair of spatial subchannels.

4.3. Performance Comparisons in Correlated Channel. Figure 12 shows the BER performance of the proposed DSA-SINR across different MCSs in uncorrelated channel 

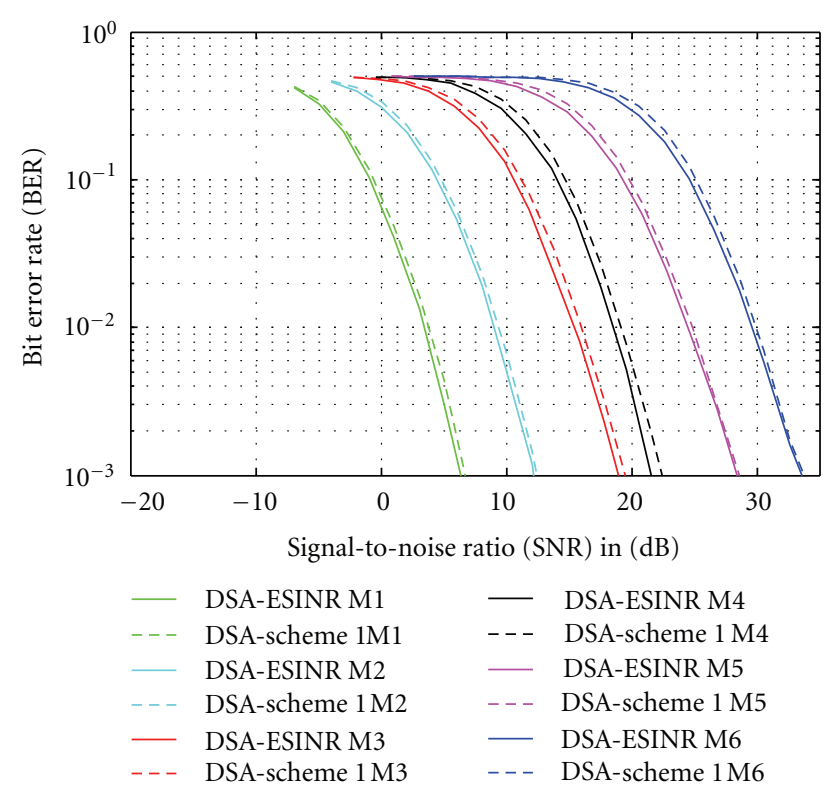

FIGURE 10: BER comparison between DSA-SINR and DSA-Scheme 1) in "Forced" uncorrelated channel.

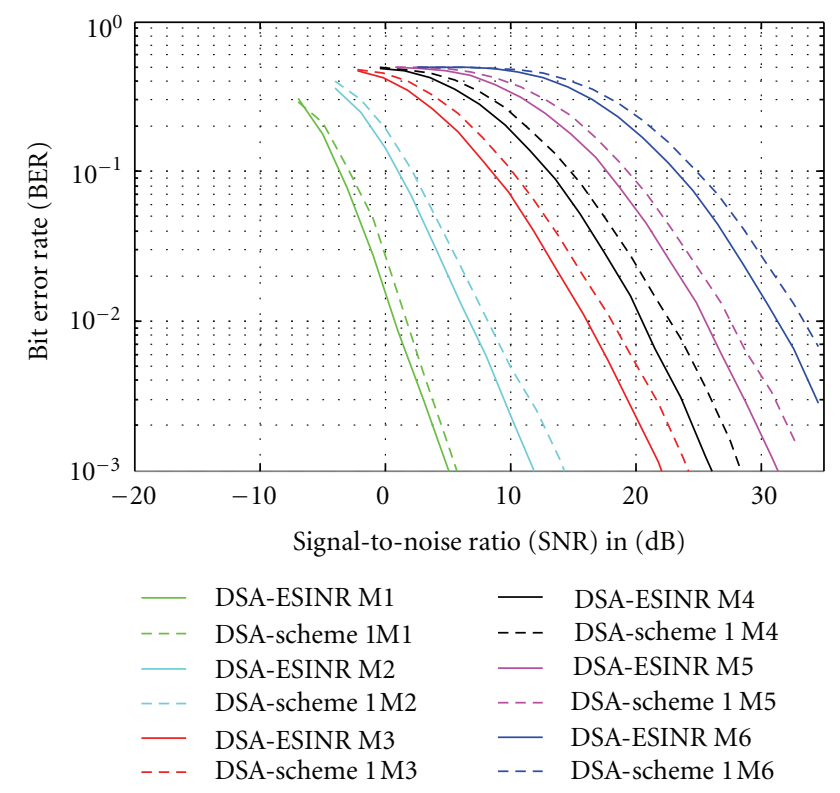

FIGURE 11: BER comparison between DSA-SINR and DSA-Scheme 1 in "Default" uncorrelated channel.

conditions, where common problems associated with wireless channel imperfections such as deep fades and ISI can be found, and Figure 13 in a "Fully" correlated channel, where the effect of self-interference dominates the channel and the effect of channel imperfections is less significant. From the results, the proposed DSA-SINR is able to offer BER gain across different levels of MCS in both types of correlation environment.

The BER degradation is uniform as the modulation order increases. This is expected since transmission in higher-order modulation comes at the cost of reduced robustness towards

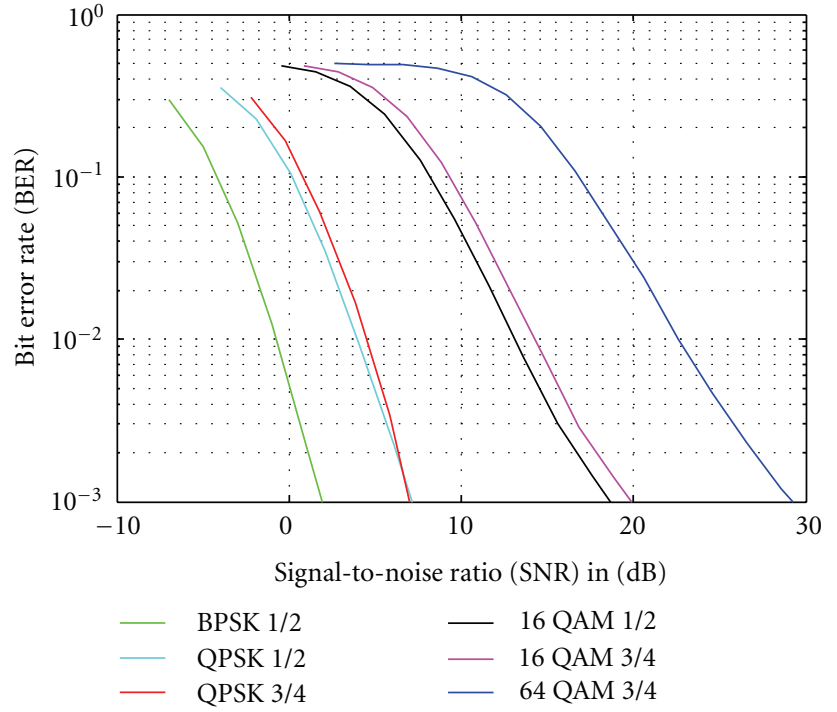

FIGURE 12: BER performance of DSA-SINR across different MCSs in uncorrelated channel.

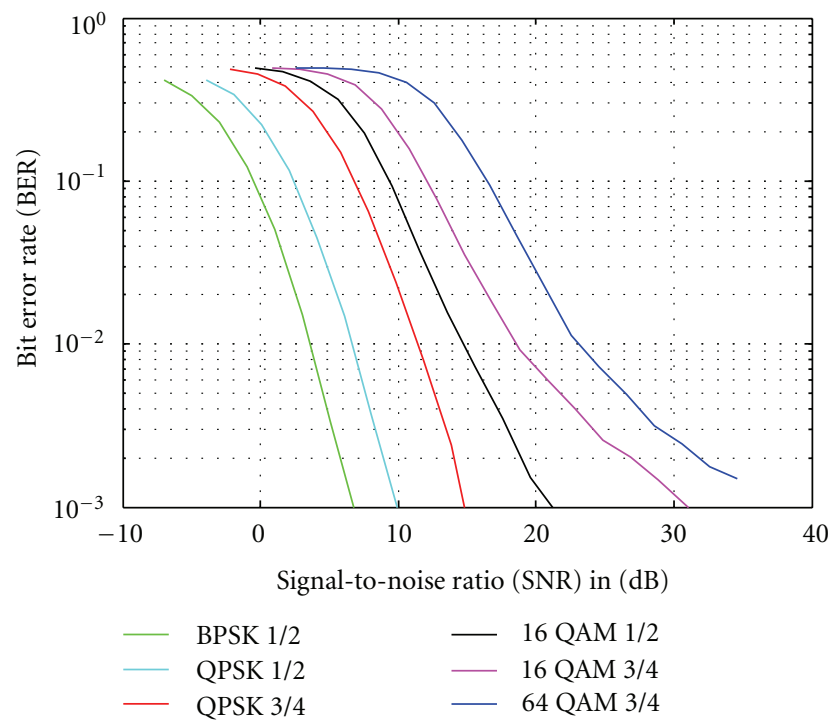

FIGURE 13: BER performance of DSA-SINR across different MCSs in a fully correlated channel.

noise and interference that is dominant in the fully correlated channel case.

In Figure 14, the performance of the proposed DSASINR is compared against that of DSA-Scheme 1 and DSAScheme 5, which have been described in Section 2.2. From the result, the benefit of DSA-SINR is apparent in a fully correlated channel. At lower SNR, DSA-SINR shares almost similar BER performance to that of the DSA-Scheme 5. The margin of BER difference is increased significantly as the SNR increases, particularly beyond $10 \mathrm{~dB}$, which implies the ability of DSA-SINR to minimise self-interference in a highly correlated channel, while DSA-Scheme 5 suffers from propagation error as the SNR increases. 


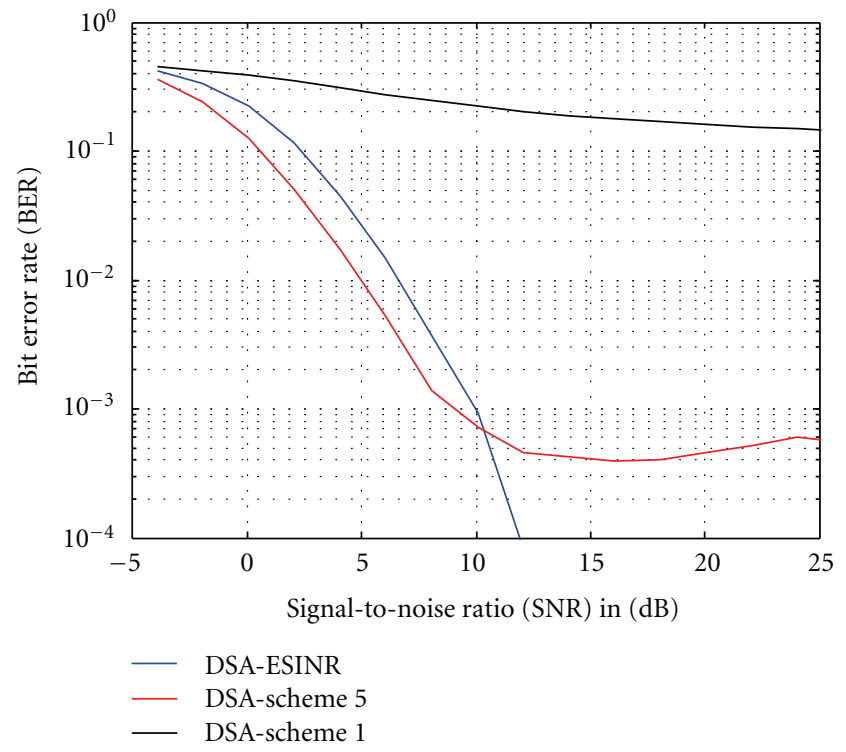

FIGURE 14: BER performance comparison for suboptimal allocation schemes in a fully correlated channel.

This is because DSA-Scheme 5 sacrifices greater degrees from the selection of the best available subcarrier in preference for avoiding allocation of the same or nearby subcarriers on different spatial subchannels, which depends on the size of $l$. In other words, there is a trade-off between mitigating self-interference and achieving diversity gain in DSA-Scheme 5.

\section{Conclusion}

This paper addresses the problem of SM downlink transmission over spatially correlated fading channels from the aspect of subcarrier allocation. A novel subcarrier allocation algorithm, known as DSA-SINR, is proposed. Design considerations of the proposed algorithm are also detailed, whereby two novel variants, known as "Partial SINR" and "Full SINR," are developed from the algorithm. From the numerical simulations and analysis, the proposed algorithm is shown to offer low complexity and to achieve substantial performance gains when operating under diverse spatial correlation cases. From the comparison analysis, "Partial SINR" showed better BER performance compared to the "Full SINR" scheme. "Partial SINR" benefited from reduced complexity since the SINR calculation is only required at the parallel subchannels.

As the spatial correlation increased in the channel, the allocation schemes were shown to reduce the effect of selfinterference, particularly DSA-SINR which had superior BER performance compared to the other allocation schemes. Error analysis also revealed the limitation of DSA-Scheme 5 in mitigating the effect of self-interference. The unit of separation on the next subcarrier parameter, denoted by $l$, is not flexible and unable to adapt to different types of correlation cases and channel model types. In general, it can be concluded that the proposed algorithm contributes towards the goal of achieving high-speed yet reliable SM transmission for future wireless networks.

\section{Acknowledgments}

The author would like to thank the National University of Malaysia for their financial support of this work, under the grant scheme UKM-GGPM-ICT-032-2011. The author also would like to thank the anonymous reviewers for their valuable feedbacks.

\section{References}

[1] S. M. Alamouti, "A simple transmit diversity technique for wireless communications," IEEE Journal on Selected Areas in Communications, vol. 16, no. 8, pp. 1451-1458, 1998.

[2] G. J. Foschini, G. D. Golden, R. A. Valenzuela, and P. W. Wolniansky, "Simplified processing for high spectral efficiency wireless communication employing multi-element arrays," IEEE Journal on Selected Areas in Communications, vol. 17, no. 11, pp. 1841-1852, 1999.

[3] G. D. Golden, C. J. Foschini, R. A. Valenzuela, and P. W. Wolniansky, "Detection algorithm and initial laboratory results using V-BLAST space-time communication architecture," Electronics Letters, vol. 35, no. 1, pp. 14-16, 1999.

[4] G. J. Foschini and M. J. Gans, "On limits of wireless communications in a fading environment when using multiple antennas," Wireless Personal Communications, vol. 6, no. 3, pp. 311-335, 1998.

[5] D. Gesbert, M. Shafi, D. S. Shiu, P. J. Smith, and A. Naguib, "From theory to practice: an overview of MIMO space-time coded wireless systems," IEEE Journal on Selected Areas in Communications, vol. 21, no. 3, pp. 281-302, 2003.

[6] W. C. Y. Lee, "Effects on correlation between two mobile radio base-station antennas," IEEE Transactions on Communications, vol. 21, no. 11, pp. 1214-1224, 1973.

[7] P. Kyritsi, D. C. Cox, R. A. Valenzuela, and P. W. Wolniansky, "Correlation analysis based on MIMO channel measurements in an indoor environment," IEEE Journal on Selected Areas in Communications, vol. 21, no. 5, pp. 713-720, 2003.

[8] M. Chamchoy, S. Promwong, P. Tangtisanon, and J. I. Takada, "Spatial correlation properties of multiantenna UWB systems for in-home scenarios," in Proceedings of the IEEE International Symposium on Communications and Information Technologies (ISCIT '04), pp. 1029-1032, October 2004.

[9] D. S. Shiu, G. J. Foschini, M. J. Gans, and J. M. Kahn, "Fading correlation and its effect on the capacity of multielement antenna systems," IEEE Transactions on Communications, vol. 48, no. 3, pp. 502-513, 2000.

[10] A. Intarapanich, P. L. Kafle, R. J. Davies, A. B. Sesay, and J. McRory, "Spatial correlation measurements for broadband MIMO wireless channels," in Proceedings of the IEEE 60th Vehicular Technology Conference (VTC '04), pp. 52-56, September 2004.

[11] K. E. Yong and C. Joohwan, "Random beamforming in MIMO systems exploiting efficient multiuser diversity," in Proceedings of the IEEE 61st Vehicular Technology Conference (VTC'05), pp. 202-205, June 2005.

[12] A. Doufexi and S. Armour, "Design considerations and physical layer performance results for a 4G OFDMA system employing dynamic subcarrier allocation," in Proceedings of the IEEE 16th International Symposium on Personal, Indoor and 
Mobile Radio Communications (PIMRC '05), pp. 357-361, September 2005.

[13] Y. Peng, Dynamic sub-carrier allocation in MIMO-OFDMA systems, Ph.D. dissertation, University of Bristol, UK, 2006.

[14] J. Jang and K. B. Lee, "Transmit power adaptation for multiuser OFDM systems," IEEE Journal on Selected Areas in Communications, vol. 21, no. 2, pp. 171-178, 2003.

[15] 3GPP, "Spatial channel model for MIMO simulations," TR 25.996 V7.0.0, 3GPP, 2007, http://www.3gpp.org/.

[16] J. P. Kermoal, L. Schumacher, K. I. Pedersen, P. E. Mogensen, and F. Frederiksen, "A stochastic MIMO radio channel model with experimental validation," IEEE Journal on Selected Areas in Communications, vol. 20, no. 6, pp. 1211-1226, 2002.

[17] K. I. Pedersen, P. E. Mogensen, and B. H. Fleury, "Spatial channel characteristics in outdoor environments and their impact on BS antenna system performance," in Proceedings of the 48th IEEE Vehicular Technology Conference (VTC'98), pp. 719-723, May 1998.

[18] K. I. Pedersen, P. E. Mogensen, and B. H. Fleury, "Power azimuth spectrum in outdoor environments," Electronics Letters, vol. 33, no. 18, pp. 1583-1584, 1997.

[19] U. Martin, "A directional radio channel model for densely built-up urban areas," in Proceedings of the 2nd European Personal Mobile Radio Conference (EPMCC '97), pp. 237-244, October 1997. 

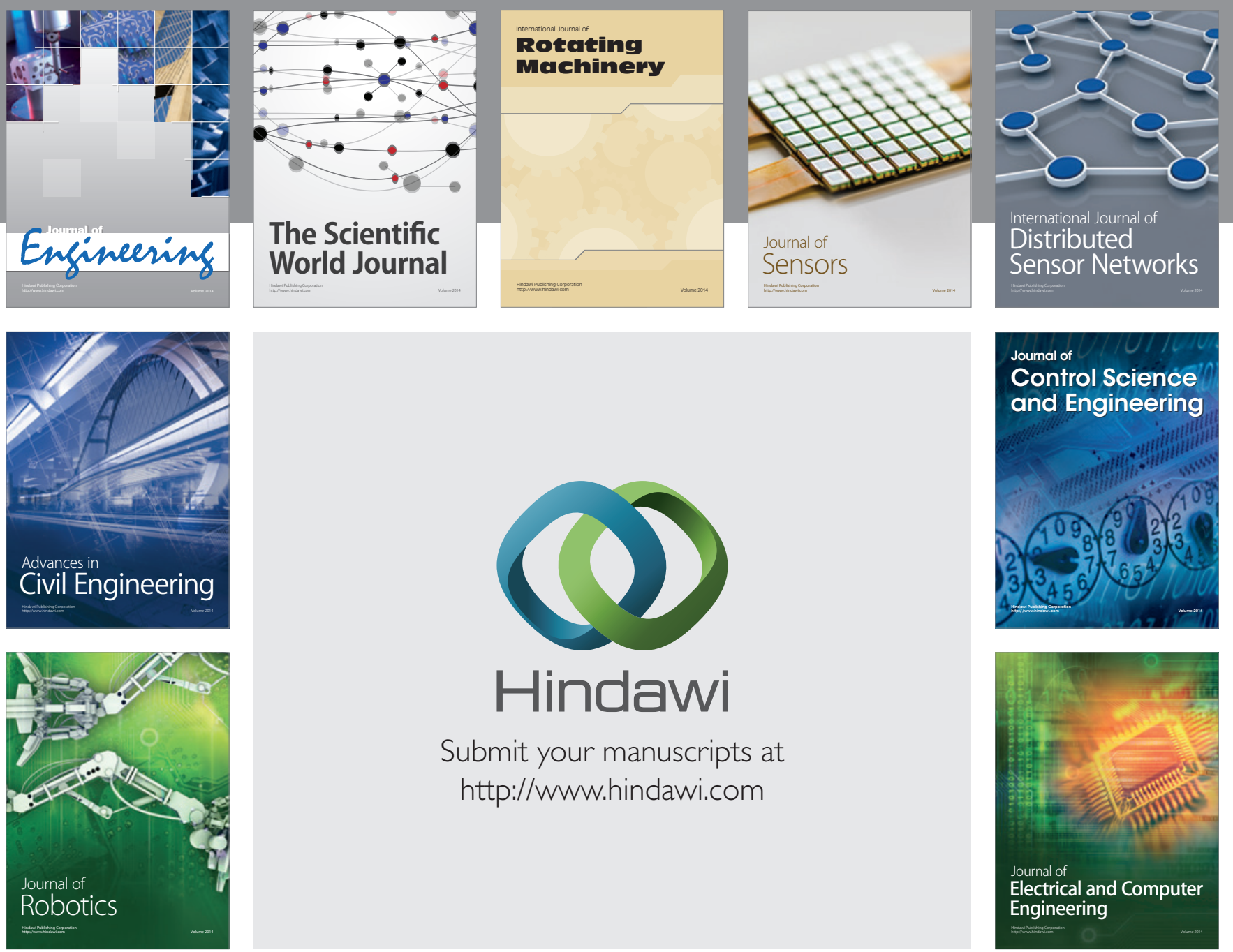

Submit your manuscripts at

http://www.hindawi.com
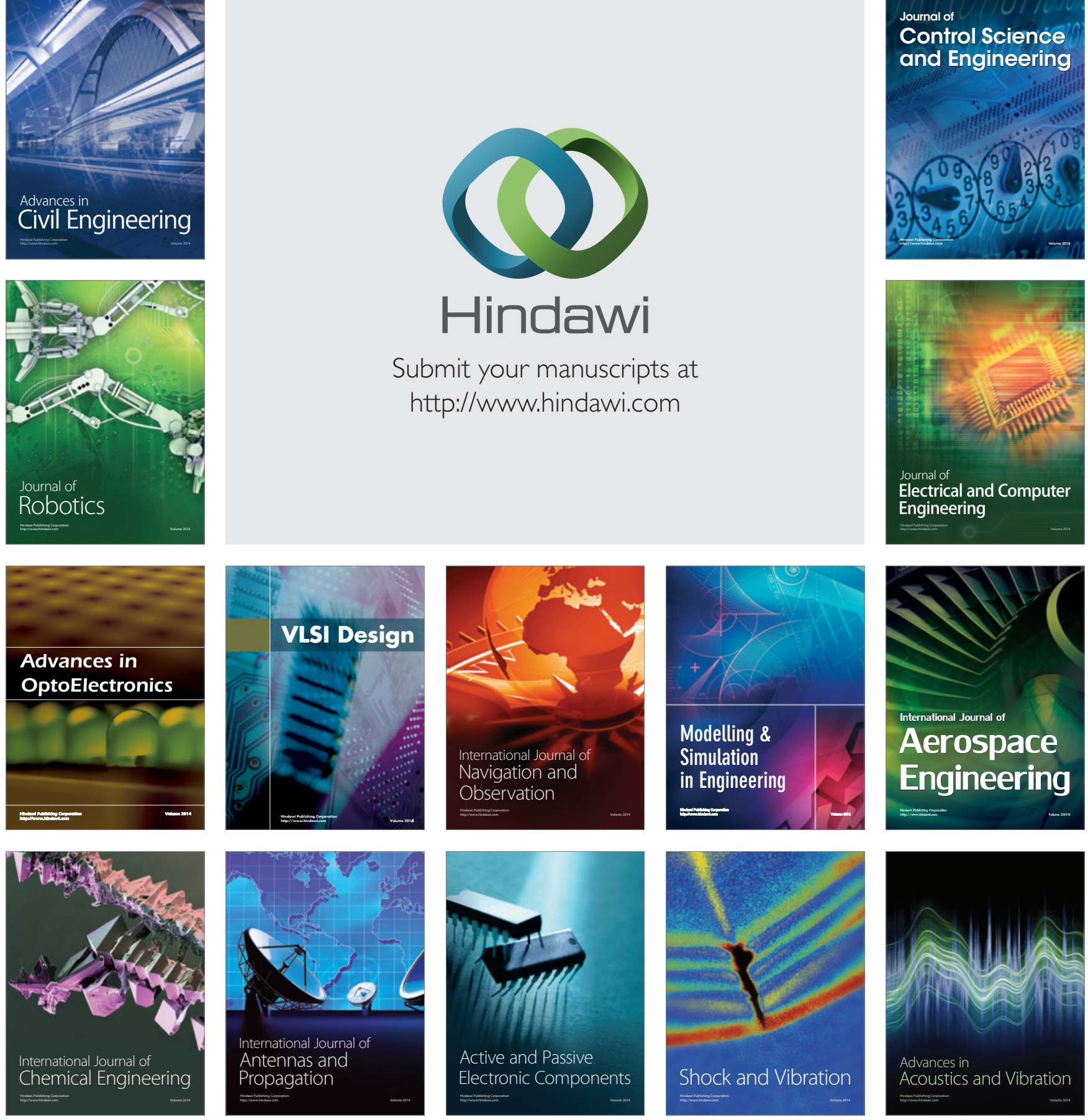